\title{
Optimizing of preoperative computed tomography for diagnosis in patients with peritoneal carcinomatosis
}

\author{
Carolin D Duhr ${ }^{1,2}$, Werner Kenn ${ }^{1}$, Ralph Kickuth ${ }^{1}$, Alexander G Kerscher ${ }^{2}$, Christoph-Thomas Germer², \\ Dietbert Hahn ${ }^{1}$ and Joerg $\mathrm{O}$ W Pelz ${ }^{2^{*}}$
}

\begin{abstract}
Background and Objective: This study evaluates whether Computer Tomography is an effective procedure for preoperative staging of patients with Peritoneal Carcinomatosis.

Method: A sample of 37 patients was analyzed with contrast enhanced abdominal Computer Tomography, followed by surgical staging. All Computer Tomography scans were evaluated 3 times by 2 radiologists with one radiologist reviewing 2 times. The efficacy of Computer Tomography was evaluated using the Spearman correlation coefficient. Correlations were analyzed by abdominopelvic region to assess results of the Peritoneal Carcinomatosis Index ( $\mathrm{PCl}$ ) aggregating the 13 regions. Surgical findings were compared to radiological findings.

Results: Results indicate high correlations between the surgical and radiological Peritoneal Carcinomatosis Indices. Analyses of the intra-class correlation between the first and second reading of one radiologist suggest high intraobserver reliability. Correlations by abdominopelvic region show higher values in the upper and middle regions and relatively lower values in the lower regions and the small bowel (correlation coefficients range between 0.418 and $0.726, p<0.010$; sensitivities range between 50\% and 96\%; and specificities range between $62 \%$ and $100 \%$ ).

Conclusion: Computer Tomography represents an effective procedure in the preoperative staging of patients with PC. However, results by abdominopelvic region show lower correlation, therefore suggest lower efficacy. These results are supported by analyses of sensitivity and accuracy by lesion size. This suggests that Computer Tomography is an effective procedure for pre-operative staging but less for determining a tumor's accurate extent.
\end{abstract}

Keywords: Carcinomatosis, $\mathrm{PCl}$, diagnosis

\section{Introduction}

Peritoneal Carcinomatosis ("PC") is a common metastasis location for many tumor variances with high incidences occurring in ovarian, gastric, and colorectal cancers. In literature, the occurrence frequencies for peritoneal metastases in ovarian, gastric, and colorectal cancers amount to $71 \%, 17 \%$, and $10 \%$, respectively $[1,2]$. Due to its natural history, PC is commonly associated with weak prognosis [3].

Today, various therapeutic procedures for PC exist with treatments being dependent on the PC's location

\footnotetext{
* Correspondence: pelz_j@klinik.uni-wuerzburg.de

${ }^{2}$ Department of General-, Visceral-, and Paediatric Surgery, University of Wuerzburg, Germany

Full list of author information is available at the end of the article
}

and extent. The existence of peritoneal disease leads to different therapeutic procedures including exclusive use of systemic chemotherapy, cytoreductive surgery combined with or without hyperthermic intra-abdominal intra-peritoneal chemotherapy or exclusive palliative management. In order to achieve a highly selective group of patients a wide array of complex therapeutic procedures constitutes the current state of clinical research, including curative focused cytoreductive surgery ("CS") and hyperthermic intraperitoneal chemoperfusion ("HIPEC") [4-12].

Elias et al. demonstrated a median survival of 5 years with a $51 \%$ survival rate which is achieved by applying the HIPEC approach in a sample of well selected patients with PC of colorectal origin [13]. To ensure 
that patients benefit from multi-modality treatment, it is mandatory to apply this treatment on patients with limited peritoneal disease where a complete cytoreduction can be achieved. Contrast enhanced abdominopelvic computed tomography ("CT") is a frequently used preoperative radiologic imaging modality to diagnose cancer within the abdominal cavity [3]. In former research the efficacy of CT diagnosing PC is not well established with an unknown size of implants being detected reliably [14]. Earlier work on CT of peritoneal malignancy was devoted to the detection of disease [15-19].

More recent studies analyzing the size of implants record varying sensitivities of $63 \%$ to $90 \%$ and specificities of up to $100 \%$ for the diagnosis of peritoneal metastasis [3]. However, in further work, sensitivities range from $41 \%$ to $79 \%$ and specificities are observed of up to $100 \%$ [20-22].

In a recent study by Coakley et al. ovarian carcinomatosis has been detected at relatively higher sensitivities of $85 \%$ to $93 \%$ [3]. Results were obtained with singlerow CT scanners. The multi-row detector CT (MDCT) technology following the single-row CT scanner technology was able to show improved sensitivities and shortened examination time by allowing the generation of thin slices with subsequent multi-planar reconstruction (MPR). This technology was able to extents and metastatic spreads in patients with various malignancies as better imaging results were achieved through improved illustration of peritoneal implants. Calculated sensitivities are dependent on the lesion size ("LS") and are relatively weak for small lesion sizes; for example, sensitivities for lesions of less than $1 \mathrm{~cm}$ were observed between $20 \%$ and $25 \%$ resulting in low reliability of diagnosis quality $[3,20,22]$.

A commonly applied regime for the quantification of the extent of PC and its deposits is the Peritoneal Cancer Index ("PCI") developed by Sugarbaker [23]. Today the PCI is acknowledged by research and used for determining therapeutic measures. It serves as an independent prognostic indicator for long-term outcomes in PC. The PCI is calculated as the sum of numerical lesion scores assigned to 13 abdominopelvic regions. The lesion score relates to the largest visible tumor deposit.

The objective of this study is to analyze the appropriateness and accuracy of $\mathrm{CT}$ as pre-operative diagnostic procedure and in combination with $\mathrm{PCI}$ as its indicator in detecting and determining the size and extent of PC.

\section{Materials and methods}

Three individual readers of two radiologists and one surgeon evaluated pre-operative contrast enhanced abdominal CT scans for a population of 37 patients. Patients in the population underwent explorative laparotomy and were suspected to have PC from primary solid tumors.

Tumor spread, localization and size were described and documented applying Sugarbaker's PCI and lesion size schemes in both, radiological as well as surgical investigations. Radiological observations were retrospectively compared to surgical observations, whereby surgical findings were regarded as the Gold Standard. Radiological findings were then statistically analyzed using correlation analyses including inter-observer as well as intra-observer reliability analyses.

\section{CT protocol}

All patients underwent $\mathrm{CT}$ scans according to a standardized CT acquisition protocol. Contrast enhancements were used including an oral contrast agent and the rectal filling Gastrolux (Sanochemia Diagnostics, Germany). All patients received intravenous injections of $110 \mathrm{~mL}$ Iomeprol (Imeron 300, Bracco Imaging, Germany) with a flow rate of $3 \mathrm{~mL} / \mathrm{s}$. A multi-slice CT scan (Siemens Somatotom Sensation 64, Germany) was used and all scans were conducted at $120 \mathrm{kV}$ with $220 \mathrm{mAs}$ as well as applying a care dose. Subsequent CT scans were started with a delay of 70 seconds. The collimation was $0.6 \mathrm{~mm}$ and the slice thickness was $5 \mathrm{~mm}$, including coronal and sagittal reformations.

\section{Patient population}

In this study a sample of 37 patients was investigated. Within this population 23 female and 14 male patients were between 24 and 78 years with an average age of 66 years at the time of the CT scan. All patients have shown solid primary tumors and underwent primary tumor surgery shortly after the CT scan (no more than 4 weeks).

In this population 32 patients had PC and 5 no PC; no patients were excluded from the study. The median time between CT scan and surgery was 10 days. Out of the 37 patients, 7 had colon cancer, 6 had gastric cancer, 5 had pseudomyxoma peritonei, 5 had ovarial cancer, 4 had pancreatic cancer, 3 had cancer of unknown primary, 2 had appendiceal cancer, and one patient each had adenocarcinoma of the abdominal wall, malignant mesenterial mesothelioma, gastrointestinal stomal tumor, mammary carcinoma, and pleural mesothelioma.

\section{System of tumor determination and description of $\mathrm{PCI}$ and LS score}

The PCI is an accepted score for the quantification of tumor spread and localization. It serves as an indicator for therapy planning and prognosis. The entire abdominal and intestinal region is divided into 13 regions (Figure 1). 

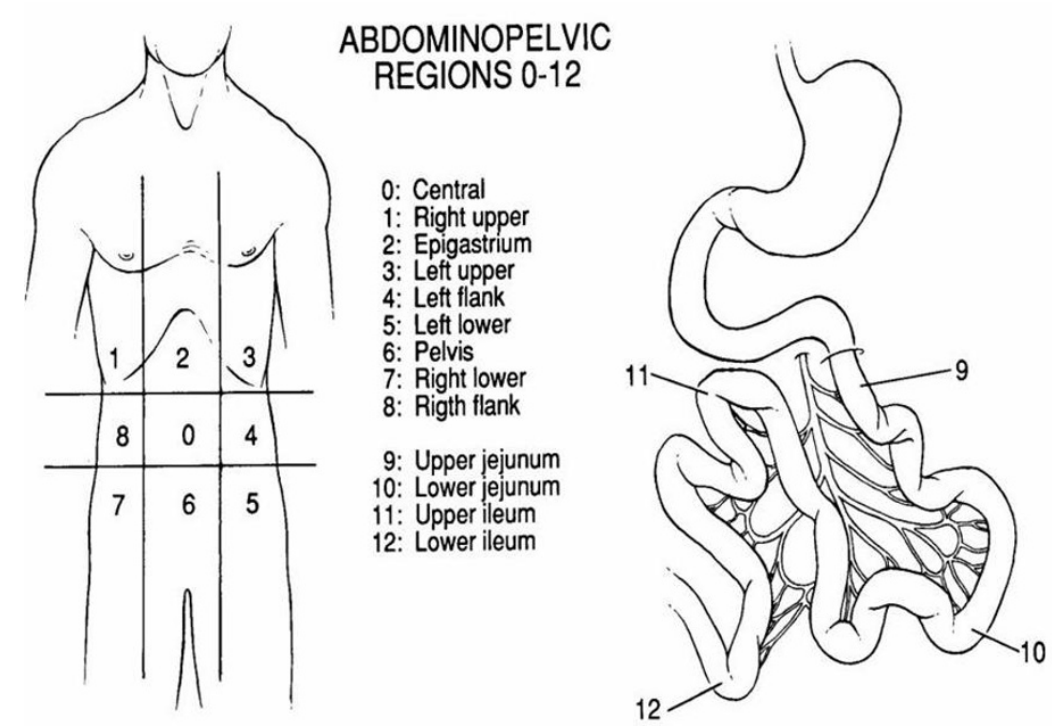

Figure 1 The Sugarbaker's Peritoneal Cancer (PCI) describes the involvement of abdominal tumor mass., [21].

In each of the 13 regions the maximum visible lesion size is measured and assigned to a lesion size score between LS $=0$ and $\mathrm{LS}=3$. LS $=0$ means no tumor visible, $\mathrm{LS}=1$ means a tumor lesion size below $0.5 \mathrm{~cm}$, $\mathrm{LS}=2$ means a tumor lesion size between $0.5 \mathrm{~cm}$ and 5 $\mathrm{cm}$, and LS $=3$ means a tumor lesion size larger than 5 $\mathrm{cm}$ or describes a confluent tumor.

LS scores in the individual regions are summed up to the PCI score which can assume a minimum score of 0 and a maximum score of 39 .

The PCI is a semi-quantitative indicator for the determination of the extent of spread of peritoneal tumor. The success of complete cytoreductive surgery and prognosis of the patient correlates with the PCI. Patients with PC of colorectal origin with a reported PCI score of less than or equal to 10 have a 5 year survival rate of $50 \%$; and lower survival rates of $20 \%$ and $0 \%$ for PCI scores between 11 and 20 , and over 20 , respectively.

\section{Radiological analyses}

CT scans were conducted before explorative laparotomy with $\mathrm{CT}$ results being compared to intra-operative findings. In total, all CT scans were evaluated three times independently with some time-lag between each evaluation reading. Two radiological senior physicians with 20 years of experience evaluated the PCI scores independently from each other to achieve inter-observer reliability. Radiologist 1 reviewed scans twice to achieve intraobserver reliability, whereas Radiologist 2 only reviewed once. In order to obtain unbiased results evaluating radiologists were neither informed about the status of primary tumor nor the PC. In addition, a control group of 5 patients was added to the study that did not show $\mathrm{PC}$ in the explorative laparotomy.

\section{Surgical analyses}

The explorative laparotomy and intra-operative data evaluations were conducted by a surgical team of one surgeon following a standard procedure and protocol. All patients underwent surgery in the same institution. Surgical and pathological findings were evaluated prospectively and documented.

In particular, the PCI score assignment was conducted by a surgical senior physician with 11 years experience in PC surgery. Intra-operative results regarding PC existence, lesion size, and localization served as Gold Standard.

\section{Statistical analyses}

Radiological and surgical PCI scores were compared to each other applying the Spearman correlation coefficient to measure inter-rater reliability. Inter-observer reliability between two radiological readings was also analyzed using the Spearman correlation coefficient. Intra-observer reliability between the two readings of the same radiologist was measured using the intra-class correlation coefficient ("ICC"). All correlations were tested for statistical significance using the p-value. Different PCI scores were also evaluated for statistical difference applying the Wilcoxon rank test. In addition to correlation coefficients, both, sensitivities and specificities were calculated for the individual abdominal regions as well as lesion size. Statistical analyses were performed using Excel software with Analyse-IT statistical package. 


\section{Results}

Differences in $\mathrm{PCl}$ results for intra-operative and radiological diagnosis

PCI observations of 37 intra-operative procedures conducted by a single surgeon are compared with independent PCI observations of two radiologists. Key findings are high levels of correlation between the intra-operative and radiological observations showing correlation coefficients of around 0.9. In particular, compared to intraoperative findings, Radiologist 1 shows correlations of 0.930 (Figure 2) and 0.888 for his first and second reading, respectively. Radiologist 2 shows a correlation of 0.887 .

All correlation coefficients in Table 1 is statistically significant at p-values below 0.001 . In order to assess the reliability of findings, the intra and inter-rater reliabilities of observations by Radiologist 1 and Radiologist 2 were examined. Results for intra-rater reliability of Radiologist 1 first and second reading show high levels of reliability indicated by an intra-class correlation coefficient ("ICC") of 0.909 (p-value < 0.001). Correlation coefficients of 0.913 and 0.953 between readings of Radiologist 1 and Radiologist 2 demonstrate high interrater reliability.

Sensitivity and specificity between Radiologist 1 (first reading) and the Gold Standard are 94\% and 80\%, respectively (Table 2). Specificities are consistent for the other two radiological readings at $80 \%$ as both radiologists correctly detected 4 patients without PC (i.e., without an intra-operative PC finding) but observed 1 false positive for the same patient without PC. However, for this fifth patient without PC, PCI scores are PCI $=2$ with 2 regions of $\mathrm{LS}=1$ for the first reading of Radiologist $1, \mathrm{PCI}=10$ with 8 regions of LS $=1$ and 1 region
Table 1 Overview of correlation coefficients.

\begin{tabular}{lrrr}
\hline & \multicolumn{2}{c}{ Radiologist 1 } & Radiologist 2 \\
\cline { 2 - 3 } & 1st Reading & 2nd Reading & \\
Intra-operative & 0,930 & 0,888 & 0,887 \\
Intra-rater Reliability: & & & \\
1st Reading Radiologist 1 & 0,909 & \\
\hline Inter-rater Reliability: & & \\
1st Reading Radiologist 1 & 0,953 & \\
\hline 2nd Reading Radiologist 1 & 0,913 \\
\hline
\end{tabular}

of LS $=2$ for the second reading of Radiologist 1 , as well as PCI $=7$ with 7 regions of LS $=1$ for the first reading of Radiologist 2 . This demonstrates that the false positives were assigned small lesion sizes. The sensitivity for the second reading of Radiologist 1 is $94 \%$ corresponding to his first reading although true positives and/or false negatives are different by one patient. Radiologist 2 shows a sensitivity of $97 \%$ (Table 2).

In most relations, the Wilcoxon rank test, used to analyze differences among the intra-operative observations and radiologist observations, indicates statistically insignificant differences at p-values > 0.05 (Table 3).

In light of high levels of correlation between intraoperative and radiological observations the insignificantly different recorded findings suggest that both radiologists evaluated the magnitude of PCI insignificantly differently than the surgeon (i.e. Gold Standard).

\section{$\mathrm{CT}$ analysis by abdominopelvic regions}

Correlation analyses by abdominopelvic region were conducted between the intra-operative findings (Gold Standard) and the first reading of Radiologist 1. Results by region show varying correlation coefficients ranging

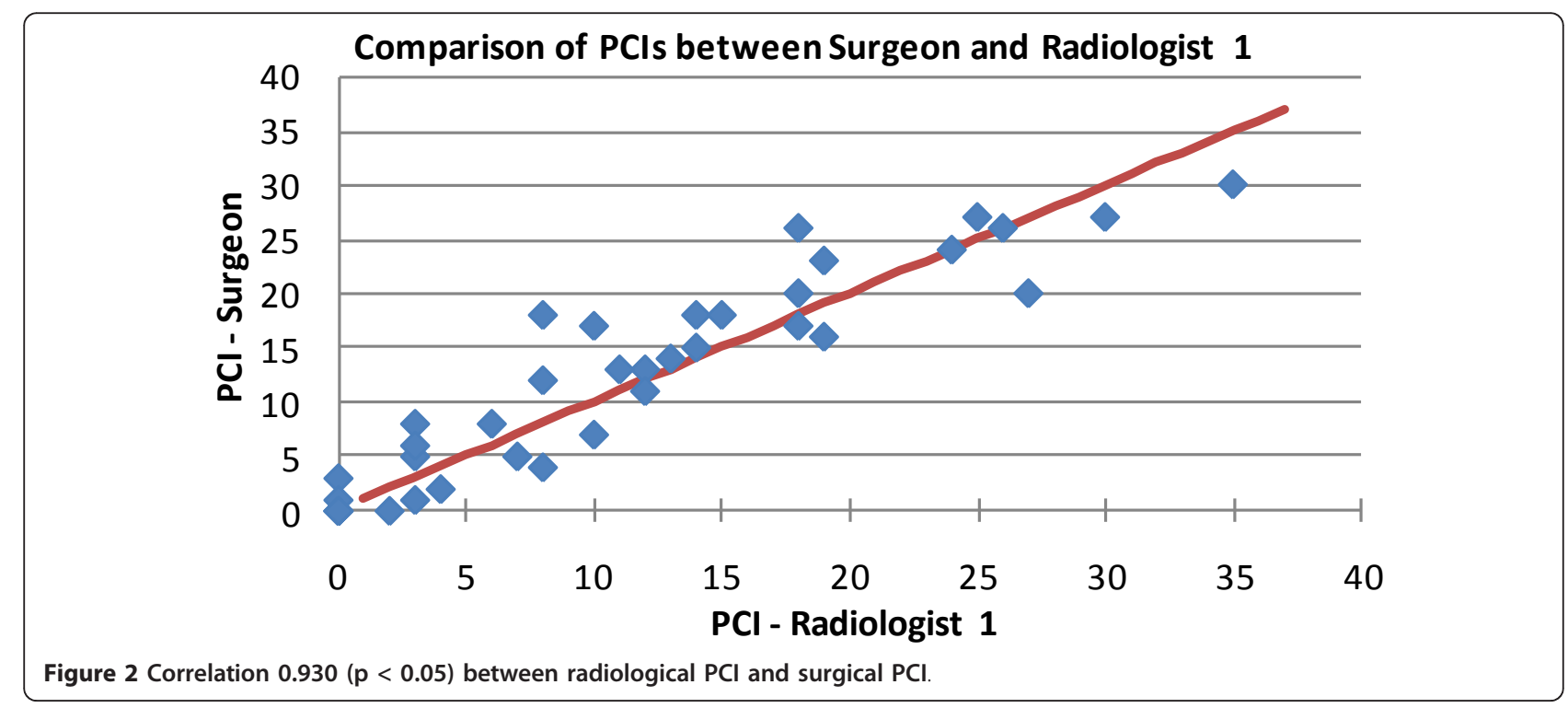


Table 2 Overview of sensitivites and specificities of Radiologist 1 and Radiologist 2.

\begin{tabular}{lrrr}
\hline & \multicolumn{2}{c}{ Radiologist 1 } & Radiologist 2 \\
\cline { 2 - 4 } True positives & 1st Reading & 2nd Reading & \\
False negatives & 30 & 30 & 31 \\
Sensitivity & 2 & 2 & 1 \\
\hline True negatives & $\mathbf{9 4 \%}$ & $\mathbf{9 4 \%}$ & $\mathbf{9 7 \%}$ \\
False positives & 4 & 4 & 4 \\
Specificity & 1 & 1 & 1 \\
PPV & $\mathbf{8 0 \%}$ & $\mathbf{8 0 \%}$ & $\mathbf{8 0 \%}$ \\
NPV & $\mathbf{9 7 \%}$ & $\mathbf{9 7 \%}$ & $\mathbf{9 7 \%}$ \\
\hline
\end{tabular}

from 0.418 to 0.881 at statistically significant levels (pvalue $<0.05$ ) (Table 4 ).

The analysis of results shows relatively higher correlations in the upper and middle abdominopelvic regions compared to the lower region and the smaller bowel. However, the upper and middle abdominopelvic regions show lower sensitivity and specificity at $86 \%$ and $73 \%$, respectively, compared to the lower abdominopelvic region. This result suggests a relatively high CT performance. CT performance in the smaller bowel is inferior with lower levels of correlation and levels of sensitivity below 0.6 and $70 \%$, respectively (Figure 3 ).

CT performance is high in the middle abdominopelvic regions which have more evenly distributed lesions sizes of 1,2 , and 3 compared to the upper region with a concentration around lesion size 1 (Table 5).

Differences in results by abdominopelvic region appear to be due to different lesion sizes (excepted for small bowel evaluation) and not because of anatomic or pathologic differences.

\section{Analysis by lesion size}

Analysis of findings by lesion size show that results depend on lesion size and that sensitivities are improved with increasing lesion sizes but matching observations (defined as the number of correct radiological tumor size observations divided through the number of total observations) show a declining rate (Table 6).

While sensitivities increase from $74 \%$ for LS $=1$ to $90 \%$ for LS $=3$ with a peak at LS $=2$ of $97 \%$, the proportion of actually matching observations between the
Gold Standard and Radiologist 1 declines from 84\% for $\mathrm{LS}=0$ to $34 \%$ for $\mathrm{LS}=3$.

\section{Discussion}

PC is a metastasis derived from different primary tumors such as the ovarian, the abdominal, and the colorectal carcinomas (in order of highest frequency). In many cases, a progressed stadium of tumor can be observed in connection with diagnosed PC and is often associated with a negative prognosis. The survival rate of patients with peritoneal metastases from colorectal origin is low with 5 to 32 months [24].

Several treatment methods exist for the therapy of PC. A multimodal treatment procedure introduced in 1990 is commonly used including cytoreductive surgery with subsequent intra-abdominal and intra-peritoneal hypothermic chemotherapy. This treatment procedure allows a 5 year survival rate of $30 \%$ to $45 \%$ [25].

The outcomes of PC diagnosis and prognosis are primarily dependent on the parameters of tumor spread, localization, and lesion size. Exact determinations of these parameters is therefore of clinical importance for determining and improving common prognosis and therapy planning. In clinical practice it is of importance to decide which therapy regime to choose, and it is furthermore important to find out if a patient has to be excluded from a certain therapy regime or not. Patients with a PCI over 20 are regarded not to be appropriate for cytoreductive surgery ("CRS").

Most studies were conducted with ovarian carcinoma with primary tumor. Further studies with patients with $\mathrm{PC}$ of non-gynecological origin such as the colorectal carcinoma would be of clinical interest.

In our study the correlation between radiological and intra-operative finding is very high with a correlation coefficient around 0.9. All correlations were statistically significant at p-values below 0.001 .

Notable are correlation coefficients of single regions that are below 0.900 and range between 0.418 and 0.881 compared to an overall PCI correlation of 0.930 . This suggests that the PCI, as summation of the observations in the 13 regions, could be misleading due to its aggregating effect. Through this summation, potential individual differences in observations could compensate each other leading to an overall lower PCI difference.

Table 3 Significance of difference in results demonstrated with the Wilcoxon rank test (p-Value).

\begin{tabular}{|c|c|c|c|}
\hline \multicolumn{4}{|c|}{ Significance of difference in results } \\
\hline & First Reading & Second Reading & Reading \\
\hline Wilcoxon rank test ( $p$-Value) & Radiologist 1 & Radiologist 1 & Radiologist 2 \\
\hline Intra-operative (surgeon) & 0,35 & 0,42 & 0,02 \\
\hline First Reading Radiologist 1 & & 0,66 & 0,02 \\
\hline Second Reading Radiologist 1 & & & 0,08 \\
\hline
\end{tabular}


Table 4 Results by abdominopelvic region including correlation coefficient, p-value, sensitivity, specificity, underestimation, and overestimation.

\begin{tabular}{|c|c|c|c|c|c|c|c|c|c|c|c|c|c|}
\hline & Abdomi & opelvic r & jion & & & & & & & & & & \\
\hline & & Upper & & & Middle & & & Lower & & & Small & owel & \\
\hline & 1 & 2 & 3 & 8 & 0 & 4 & 7 & 6 & 5 & 9 & 10 & 11 & 12 \\
\hline Correlation & 0,748 & 0,713 & 0,660 & 0,823 & 0,774 & 0,881 & 0,659 & 0,726 & 0,698 & 0,418 & 0,431 & 0,519 & 0,533 \\
\hline$P$-value & 0,000 & 0,000 & 0,000 & 0,000 & 0,000 & 0,000 & 0,000 & 0,000 & 0,000 & 0,010 & 0,008 & 0,001 & 0,001 \\
\hline Sensitivity & $86 \%$ & $86 \%$ & $91 \%$ & $96 \%$ & $86 \%$ & $100 \%$ & $96 \%$ & $88 \%$ & $93 \%$ & $50 \%$ & $53 \%$ & $70 \%$ & $70 \%$ \\
\hline Specificity & $87 \%$ & $75 \%$ & $73 \%$ & $79 \%$ & $75 \%$ & $94 \%$ & $62 \%$ & $69 \%$ & $80 \%$ & $89 \%$ & $94 \%$ & $100 \%$ & $100 \%$ \\
\hline Under estimated & $14 \%$ & $14 \%$ & $9 \%$ & $4 \%$ & $14 \%$ & $0 \%$ & $4 \%$ & $13 \%$ & $7 \%$ & $50 \%$ & $47 \%$ & $30 \%$ & $30 \%$ \\
\hline Over estimated & $13 \%$ & $25 \%$ & $27 \%$ & $21 \%$ & $25 \%$ & $6 \%$ & $38 \%$ & $31 \%$ & $20 \%$ & $11 \%$ & $6 \%$ & $0 \%$ & $0 \%$ \\
\hline
\end{tabular}

The distribution of lesion sizes in the various abdominopelvic regions is shown in Table 5.

Evaluations of the PCI Scores are depending on the expertise and experience of the radiologist and surgeon. For this reason another independent reading of the CT scans by a second radiologist was included in this study. We measured a high inter rater reliability between both radiologists of 0.953 and 0.913 at $\mathrm{p}$ values below 0.001 .

Furthermore, to assess the reliability of findings one radiologist evaluated CT scans a second time. The intrarater reliability supported by in the intra class coefficient amounted to 0.909 ( $\mathrm{p}<0.001$ ) which could be considered very high.

This study shows that both radiologists with a similar background of experience evaluated CT scans with high levels of correlation. These finding are in line with other recent published studies.

In a study by Coakley et al. in which preoperative CT scans of patients with ovarian carcinoma were reviewed by three independent readers, showed that the depiction of peritoneal metastases is good to excellent [3]. The Kappa value in his study ranged between 0.75 to 0.91 which indicates good to excellent inter-observer agreements.

This study shows an overall sensitivity of $94 \%$ and specificity of $80 \%$ which are broadly in line with more recent studies. Studies by Coakley et al. and Tempany et al. showed sensitivities of $85 \%$ to $93 \%$ in the detection of peritoneal metastases in patients with ovarian cancer $[3,26]$.

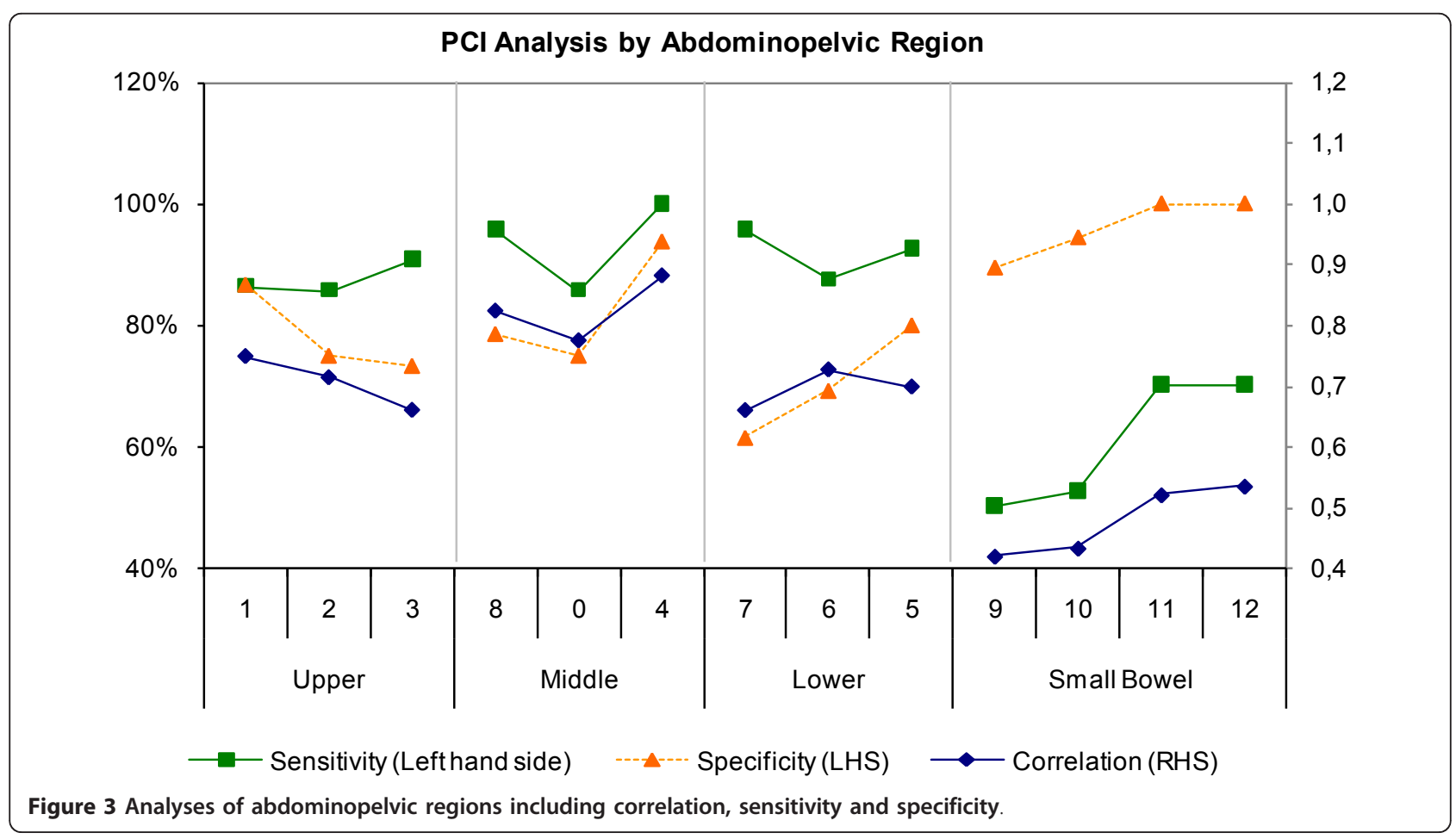


Table 5 Distribution of lesion sizes in the various abdominopelvic regions.

\begin{tabular}{|c|c|c|c|c|c|c|c|c|c|c|c|c|c|}
\hline \multirow[b]{3}{*}{ Lesion size } & \multicolumn{13}{|c|}{ Abdominopelvic region } \\
\hline & \multicolumn{3}{|c|}{ Upper } & \multicolumn{3}{|c|}{ Middle } & \multicolumn{3}{|c|}{ Lower } & \multicolumn{4}{|c|}{ Small Bowel } \\
\hline & 1 & 2 & 3 & 8 & 0 & 4 & 7 & 6 & 5 & 9 & 10 & 11 & 12 \\
\hline $\mathrm{LS}=0$ & 15 & 16 & 15 & 14 & 16 & 16 & 13 & 13 & 10 & 19 & 18 & 17 & 17 \\
\hline $\mathrm{LS}=1$ & 11 & 12 & 14 & 8 & 5 & 10 & 9 & 10 & 15 & 14 & 16 & 18 & 17 \\
\hline $\mathrm{LS}=2$ & 6 & 4 & 4 & 8 & 7 & 7 & 11 & 11 & 7 & 3 & 2 & 1 & 2 \\
\hline $\mathrm{LS}=3$ & 5 & 5 & 4 & 7 & 9 & 4 & 4 & 3 & 5 & 1 & 1 & 1 & 1 \\
\hline $\mathrm{LS}=0$ & $41 \%$ & $43 \%$ & $41 \%$ & $38 \%$ & $43 \%$ & $43 \%$ & $35 \%$ & $35 \%$ & $27 \%$ & $51 \%$ & $49 \%$ & $46 \%$ & $46 \%$ \\
\hline $\mathrm{LS}=1$ & $30 \%$ & $32 \%$ & $38 \%$ & $22 \%$ & $14 \%$ & $27 \%$ & $24 \%$ & $27 \%$ & $41 \%$ & $38 \%$ & $43 \%$ & $49 \%$ & $46 \%$ \\
\hline $\mathrm{LS}=2$ & $16 \%$ & $11 \%$ & $11 \%$ & $22 \%$ & $19 \%$ & $19 \%$ & $30 \%$ & $30 \%$ & $19 \%$ & $8 \%$ & $5 \%$ & $3 \%$ & $5 \%$ \\
\hline $\mathrm{LS}=3$ & $14 \%$ & $14 \%$ & $11 \%$ & $19 \%$ & $24 \%$ & $11 \%$ & $11 \%$ & $8 \%$ & $14 \%$ & $3 \%$ & $3 \%$ & $3 \%$ & $3 \%$ \\
\hline
\end{tabular}

Correlation was analyzed by abdominopelvic regions ranging from 0.418 to 0.881 at statistically significant levels. Higher correlations were found in upper and middle abdominopelvic regions compared to lower region and small bowel. Upper and middle abdominopelvic regions show higher sensitivities and specificities above $86 \%$ and $73 \%$, respectively, compared to the lower region.

The best evaluated regions were left flank (4) with $100 \%$ sensitivity, right lower (7) and right flank (8) with $96 \%$ and left lower (5) with $93 \%$. The inclusion of the smaller bowel plays an important part in the prognosis and a reason for including it in this study regarding tumor extent and size. In line with finding in previous studies, CT is less appropriate for diagnosing the small bowel region. In this region, results show the lowest sensitivities in this study of $50 \%$ to $70 \%$ and correlation levels between 0.418 and 0.533 . The study by Koh et al. shows sensitivities of $8 \%$ to $14 \%$ in the small bowel region which can also be found in a study by de Bree et al. [4,14]. The improvement of sensitivities in this study is expected to be a result of the application of a single center setup with a highly standardized CT acquisition protocol.

Table 6 Analysis of findings by lesion size demonstrating true positives, false negatives, sensitivity, specificity, accuracy, underestimation, overestimation.

\begin{tabular}{|c|c|c|c|c|}
\hline & $\underline{L S}=0$ & $\underline{L S}=1$ & $\underline{L S}=2$ & $\underline{L S}=3$ \\
\hline Region count & 199 & 159 & 73 & 50 \\
\hline True positives & n.a. & 118 & 71 & 45 \\
\hline False negatives & n.a. & 41 & 2 & 5 \\
\hline Sensitivity & n.a. & $74 \%$ & $97 \%$ & $90 \%$ \\
\hline Specificity & $84 \%$ & n.a. & n.a. & n.a. \\
\hline Accuracy & $84 \%$ & $53 \%$ & $48 \%$ & $34 \%$ \\
\hline Under estimate & n.a. & $26 \%$ & $34 \%$ & $66 \%$ \\
\hline Over estimate & $16 \%$ & $21 \%$ & $18 \%$ & n.a. \\
\hline
\end{tabular}

$n=481$ (37 patients $\times 13$ abdominopelvic regions)

n.a. $=$ not available
Furthermore CT scans in this study were performed with oral and intravenous fillings and reconstruction. It seems probable that a standardized CT acquisition protocol leads to better results in detection rates. A possible reason for low sensitivity in the detection of PC diseases could be the time lag between the CT scan and the operative surgery during which period a tumor can potentially grow. These results observations are supported by high correlations between radiological and surgical findings and high levels of inter and intra-rater reliability.

The relatively lower performance of $\mathrm{CT}$ in the upper region can be explained by a concentration of lesions sizes of 1 where CT shows a low sensitivity. Low sensitivity and high specificity in the small bowel could stem from a high concentration of lesion sizes of 1 and 0 , respectively.

It is shown that the PCI score is depending on lesion size. With increasing lesion size the sensitivity is improving; however, accurate observations are declining with increasing lesion size. Sensitivity increases from $74 \%$ for LS $=1$ to $90 \%$ for LS $=3$, while the accuracy between surgical and radiological observations is declining from $84 \%$ for LS $=1$ to $34 \%$ for LS $=3$. A declining accuracy with an increasing lesion size is explained by increasing underestimation by the radiologist. It is observed that the radiologist systematically underestimates the Gold Standard which is supported by the findings according to lesion size.

There exist several methods for imaging PC including $\mathrm{CT}$, magnet resonance tomography, FDG-PET-Observation, or ultrasound. The most used and commonly applied method is the CT based diagnosis. Subject of this study is the assessment of appropriateness and accuracy of CT for the detection and diagnosis of PC.

Due to comparatively higher availability, lower costs, and evaluation artifacts of bowel peristaltic and breathing, CT with oral or intravenous contrast injection is still the most commonly used method for the diagnosis of PC [27]. 
In studies from 1985 until 2009 evaluating the appropriateness of CT for the detection of PC, sensitivities reach from $14 \%$ to $93 \%$ and specificities from $54 \%$ to $100 \%$. Such spectrums are expected to be the result of the advent of new technology including the multi-detector-CT or the multimodal reconstruction. The recent study by Coakley et al. showed sensitivities of $85 \%$ to 93\% and specificities of $91 \%$ to $96 \%$ for CT based PC diagnosis [3].

The increasing improvement of results could be due to the further development of technology such as the contrast enhanced spiral CT. In a study by Jacquet et al. sensitivities were first described in relation to tumor lesion size; sensitivities were recorded at $70 \%$ for a maximum lesion size of $2 \mathrm{~cm}$ whereas at $28 \%$ for lesion sizes of less than $0.5 \mathrm{~cm} \mathrm{[22].}$

Coakley et al. investigated sensitivities in relation to lesion size and showed $25 \%$ to $50 \%$ lower sensitivities for lesion sizes below $1 \mathrm{~cm}$ compared to overall observed sensitivities between $85 \%$ and $93 \%$, thereby suggesting lower performance of $\mathrm{CT}$ for small lesion sizes [3]. Further studies investigating CT performance for small lesion sizes would be of clinical interest.

The study by Tempany et al. investigated sensitivities in relation to origin of primary tumor; sensitivity of $92 \%$ was observed with patients with ovarian carcinoma [26]. Similarly, the study by de Bree et al. showed a sensitivity of $60 \%$ to $76 \%$ for patients with appendenix and colorectal carcinoma [20]. In most studies sensitivities were below $20 \%$ for the regions of mesenterium and small bowel [14]. According to de Bree et al. the surface can best be observed with the existence of ascites [20]. In this study the existence of ascites did not explain significantly better results.

With the introduction of more advanced technologies imaging quality has been improved. Coronary and sagittal imaging methods result in the detection of smaller tumor lesion sizes with smaller artifacts [28]. With the advent of higher resolutions of scanner improved depiction of PC could expected.

A disadvantage of CT is the distinguishing of the tumor scar tissue from the post-operative scar tissue which is almost impossible with current sophistication of CT technology.

A study by Franiel et al. investigated the impact of thinner layers and multi-planar reconstruction in connection with the observation of PC [29]. Layers of $5 \mathrm{~mm}$ thickness were found to be sufficient whereas layers of 1 $\mathrm{mm}$ in connection with MPR could improve sensitivities and diagnostic confidence. There, results were dependent on the radiologist's years of experience. A radiologist with over 10 years of experience, using 0 coronal and sagittal MPRs, could achieve an improved sensitivity of $96 \%$ when using $1 \mathrm{~mm}$ thin slices compared to $86 \%$ when using $0.5 \mathrm{~cm}$ thin slices. Best results with sensitivities of up to $100 \%$ could be shown by using MPRs.

MRI and spiral CT show similar accuracy in the detection of PC and PC size, spread and localization compared to CT. In a study by Kim et al. it is showed that MRI achieves a sensitivity of 95\% [30]. The mandatory long hold of breath proves to be difficult for many patients with tumors due to their generally weak condition. This could lead to evaluation artifacts and diminish the quality of MRI depiction [30]. Another disadvantage is the existence of ascites which could also lead to artifacts using MRI and could often be the reason for high wrong positive-rates [30].

Tempany et al. in his study investigated MRI in comparison to CT wherein higher sensitivities for MRI of 95\% compared to $92 \%$ for CT could be shown [26]. However, specificities were lower at $80 \%$ for MRI compared to $82 \%$ for CT. Overall the accuracy was not significantly different between the two modalities. Although MRI performed better at sensitivities between $85 \%$ to $90 \%$ for small modular peritoneal changes of less than $1 \mathrm{~cm}$ compared to CT with $25 \%$ to $50 \%$ it could questionable whether MRI is a superior imaging method given its relatively longer duration of observation and lower availability. In previous literature, the sensitivity of MRI for the detection of PC is approximately $85 \%$ $[31,32]$ and specificity of around $80 \%[26]$.

The appropriateness and effectiveness of PET-CT for the diagnosis of PC has yet to be investigated. The study by Dromain et al. concludes that neither CT nor PETCT could be used stand-alone for the prognosis and planning of PC therapy due to a correct detection evaluation of $70 \%$ for CT and 80\% for PET-CT [33].

The FDG-PET (2-Fluor-2-deoxy-D-Glucose positron emission tomography) is another alternative method but showed low specificities between $54 \%$ and $86 \%[34,35]$. FDG-PET also shows lower accuracy for the detection of smaller lesion sizes compared to MRT und CT [36].

The laparotomy is still the preferred method for the exact staging of investigation with histological biopsy. It is, however, also associated with peri-operative dissemination of maligned cells. Furthermore, the invasive procedure is often associated with higher rates of mortality and morbidity [37-39].

Laparoscopy is also an effective method for staging peritoneal carcinomatosis. Unfortunately, not every region can be assessed. Therefore, laparoscopy appears to be a highly suitable method of diagnosis for the small bowel in cases of vague involvement.

In line with previous research, this study underpins that $\mathrm{CT}$ is an effective imaging modality for the pre and postoperative staging of patients. A standardized CT acquisition protocol in a certified PC center and readings performed by highly experienced radiologists could 
result in sufficiently high sensitivity and specificity rates for an effective patient selection process. Relatively high sensitivities and specificities results are achieved by abdominopelvic region as well as by lesion size. However, the appropriateness of CT for the evaluation of the small bowel region is still insufficient and needs to be further investigated.

\section{Conclusion}

A standardized CT acquisition protocol in a certified peritoneal cancer center and readings performed by highly experienced radiologists seem to achieve higher values for sensitivity and specificity and therefore are expected to result in a more effective patient selection process.

\section{Author details \\ ${ }^{1}$ Department of Radiology, University of Wuerzburg, Germany. ${ }^{2}$ Department of General-, Visceral-, and Paediatric Surgery, University of Wuerzburg, Germany.}

\section{Authors' contributions}

CCD participated in the design, acquisition, analysis and interpretation of data, drafting manuscript, critical revision. WK carried out the first radiologist reading. RK carried out the second radiologist reading. AK participated in drafting and editing of manuscript, data analysis. CTG participated in the acquisition of data, critical revision. DH carried out in the data analysis and Critical revision. JOWP participated in the design, acquisition, analysis and interpretation of data, drafting manuscript, critical revision. All the authors participated in the conception, the design, data collection and interpretation, manuscript preparation, and literature search. All authors have read and approved the final manuscript.

\section{Competing interests}

The authors declare that they have no competing interests.

Received: 1 August 2011 Accepted: 21 December 2011 Published: 21 December 2011

\section{References}

1. Buy JN, Moss AA, Ghossain MA Sciot C, Malbec L, Vadrot D, Paniel BJ, Decroix Y: Peritoneal implants from ovarian tumors: CT findings. Radiology 1988, 169:691-4.

2. Davies J, Chalmers AG, Sue-Ling HM May J, Miller GV, Martin IG, Johnston D: Spiral computed tomography and operative staging of gastric carcinoma: a comparison with histopathological staging. Gut 1997, 41:314-19

3. Coakley FV, Choi PH, Gougoutas CA Pothuri B, Venkatraman E, Chi D, Bergman A, Hricak H: Peritoneal metastases: detection with spiral CT in patients with ovarian cancer. Radiology 2002, 223:495-9.

4. de Bree E, Witkamp AJ, Zoetmulder FAN: Intraperitoneal chemotherapy for colorectal cancer. J Surg Oncol 2002, 79:46-61.

5. Witkamp AJ, de Bree E, van Goethem A, Zoetmulder FA: Rationale and techniques of intra-operative hyperthermic intraperitoneal chemotherapy. Cancer Treat Rev 2001, 27:365-74.

6. Witkamp AJ, de Bree E, Kaag MM, Boot H, Beijnen JH, van Slooten GW, van Coevorden F, Zoetmulder FA: Extensive cytoreductive surgery followed by intra-operative hyperthermic intraperitoneal chemotherapy with mitomycin-C in patients with peritoneal carcinomatosis of colorectal origin. Eur J Cancer 2001, 37:979-84

7. Verwaal VJ, van Ruth S, de Bree E, van Sloothen GW, van Tinteren H, Boot $\mathrm{H}$, Zoetmulder FA: Randomized trial of cytoreduction and hyperthermic intraperitoneal chemotherapy versus systemic chemotherapy and palliative surgery in patients with peritoneal carcinomatosis of colorectal cancer. J Clin Oncol 2003, 21:3737-43.
8. Sugarbaker PH: Management of peritoneal-surface malignancy: The surgeon's role. Langenbeck's Arch Surg 1999, 384:576-87.

9. Sugarbaker PH, Chang D: Results of treatment of 385 patients with peritoneal surface spread of appendiceal malignancy. Ann Surg Oncol 1999, 6:727-31

10. Pilati $P$, Mocellin $S$, Rossi $C R$, Foletto M, Campana L, Nitti D, Lise M: Cytoreductive surgery combined with hyperthermic intraperitoneal intraoperative chemotherapy for peritoneal carcinomatosis arising from colon adenocarcinoma. Ann Surg Oncol 2003, 10:508-13.

11. Culliford AT IV, Brooks AD, Sharma S, Saltz LB, Schwartz GK, O'Reilly EM, Ilson DH, Kemeny NE, Kelsen DP, Guillem JG, Wong WD, Cohen AM, Paty PB: Surgical debulking and intraperitoneal chemotherapy for established peritoneal metastases from colon and appendix cancer. Ann Surg Oncol 2001, 8:787-95.

12. McQuellon RP, Loggie BW, Lehman AB, Russell GB, Fleming RA, Shen P, Levine EA: Long-term survivorship and quality of life after cytoreductive surgery plus intraperitoneal hyperthermic chemotherapy for peritoneal carcinomatosis. Ann Surg Oncol 2003, 10:155-62.

13. Elias D, Lefevre JH, Chevalier J, Brouquet A, Marchal F, Classe JM, Ferron G, Guilloit JM, Meeus P, Goéré D, Bonastre J: Complete cytoreductive surgery plus intraperitoneal chemohyperthermia with oxaliplatin for peritoneal carcinomatosis of colorectal origin. J Clin Oncol 2009, 27:681-685.

14. Koh JL, Yan TD, Glenn D, Morris DL: Evaluation of preoperative computed tomography in estimating peritoneal cancer index in colorectal peritoneal carcinomatosis. Ann Surg Oncol 2009, 16:327-33.

15. Walkey MM, Friedman AC, Sohotra P, Radecki PD: CT manifestations of peritoneal carcinomatosis. AJR 1988, 150:1035-41.

16. Jeffrey RB: CT demonstration of peritoneal implants. AJR 1988, 135:323-6.

17. Seshul MB, Coulam CM: Pseudomyxoma peritonei: computed tomography and sonogram. AJR 1981, 136:803-6.

18. Dachman AH, Lichtenstein JE, Friedman AC: Mucocele of the appendix and pseudomyxoma peritonei. AJR 1985, 144:923-9.

19. Clarke-Pearson DL, Bondy LC, Dudzinski MD: Computed tomography in evaluation of patients with ovarian carcinoma in complete clinical remission. JAMA 1986, 255(6):627-30.

20. de Bree E, Koops W, Kroger R, van Ruth S, Witkamp AJ, Zoetmulder FA: Peritoneal carcinomatosis from colorectal or appendiceal origin: correlation of preoperative CT with intraoperative findings and evaluation of interobserver agreement. J Surg Oncol 2004, 86:64-73.

21. Halverson RA Jr, Panushka C, Coakley GJ, Letourneau JG, Adcock LL: Intraperitoneal contrast material improves the $\mathrm{CT}$ detection of peritoneal metastases. AJR 1991, 157:37-40.

22. Jacquet $P$, Jelinek JS, Steves MA, Sugarbaker PH: Evaluation of computed tomography in patients with peritoneal carcinomatosis. Cancer 1993, 72:1631-36.

23. Jacquet $\mathrm{P}$, Sugarbaker $\mathrm{PH}$ : Clinical research methodologies in diagnosis and staging of patients with peritoneal carcinomatosis.Edited by: Sugarbaker PH. Peritoneal Carcinomatosis: Principles of Management. Boston: Kluwer 1996; 359-74.

24. Chu DZ, Lang NP, Thompson C, Osteen PK, Westbrook KC: Peritoneal carcinomatosis in nongynecologic malignancy. Cancer 1989, 63:364-7.

25. Sadeghi B, Arvieux C, Glehen O, Beaujard AC, Rivoire M, Baulieux J, Fontaumard E, Brachet A, Caillot JL, Faure JL, Porcheron J, Peix JL, François Y, Vignal J, Gilly FN: Peritoneal carcinomatosis from nongynecologic malignancies. Results of EVOCAPE 1 multicentric prospective study. Cancer 2000, 88:358-63.

26. Tempany CMC, Zou KH, Silverman SG, Brown DL, Kurtz AB, McNeil BJ Staging of advanced ovarian cancer: comparison of imaging modalitiesreport from the Radiological Diagnostic Oncology Group. Radiology 2000, 215:761-67.

27. Raptopoulos V, Gourtsoyiannis N: Peritoneal carcinomatosis. Eur Radiol 2001, 11:2195-206.

28. Pannu HK, Bristow RE, Montz FJ, Fishman EK: Multidetector CT of peritoneal carcinomatosis from ovarian cancer. Radiographics 2003, 23:687-701.

29. Franiel T, Diederichs G, Engelken F, Elgeti T, Rost J, Rogalla P: Multidetector $\mathrm{CT}$ in peritoneal carcinomatosis: diagnostic role of thin slices and multiplanar reconstructions. Abdominal Imaging 2009, 34:49-54.

30. Kim CK, Park BK: Comparison of the MRI and integrated PET/CT findings in the preoperative detection of peritoneal carcinomatosis arising from primary ovarian cancer. J Korean Soc Radiol 2009, 60:117-26. 
31. Low RN, Barone RM, Lacey C, Sigeti JS, Alzate GD, Sebrechts CP: Peritoneal tumor: MR imaging with dilute oral barium and intravenous gadoliniumcontaining contrast agents compared with unenhanced MR imaging and CT. Radiology 1997, 204:513-20.

32. Low RN: MR imaging of the peritoneal spread of malignancy. Abdom Imaging 2007, 32:267-83.

33. Dromain C, Leboulleux S, Auperin A, Goere D, Malka D, Lumbroso J, Schumberger M, Sigal R, Elias D: Staging of peritoneal carcinomatosis: enhanced CT vs. PET/CT. Abdom Imaging 2008, 33:87-93.

34. Hubner KF, McDonald TW, Niethammer JG, Smith GT, Gould HR, Buonocore E: Assessment of primary and metastatic ovarian cancer by positron emission tomography (PET) using 2-[18F]deoxyglucose (2-[18F] FDG). Gynecol Oncol 1993, 51:197-204.

35. Romer W, Avril N, Dose J, Ziegler S, Kuhn W, Herz M, Jänicke F, Schwaiger M: Metabolic characterization of ovarian tumors with positron-emission tomography and F-18 fluorodeoxyglucose. Rofo 1997, 166:62-8.

36. Kubik-Huch RA, Doerffler W, von Schulthess GK, Marincek B, Köchli OR, Seifert B, Haller U, Steinert HC: Value of (18F)-PDG positron emission tomography, computed tomography, and magnetic resonance imaging in diagnosing primary and recurrent ovarian carcinoma. Eur Radiol 2000, 10:761-67.

37. Ketcham AS, Hoye RC, Pilch YH, Morton DL: Delayed intestinal obstruction following treatment for cancer. Cancer 1970, 25:406-10.

38. Targazona EM, Martinez J, Nadal A, Balagué C, Cardesa A, Pascual S, Trias M: Cancer dissemination during laparoscopic surgery. World J Surg 1998, 22:55-60.

39. Sheperd NA, Baxter KJ, Loves SB: Influence of local peritoneal involvment on pelvic recurrence and prognosis in rectal cancer. J Clin Pathol 1995, 48:849-55.

doi:10.1186/1477-7819-9-171

Cite this article as: Duhr et al:: Optimizing of preoperative computed tomography for diagnosis in patients with peritoneal carcinomatosis. World Journal of Surgical Oncology 2011 9:171.

\section{Submit your next manuscript to BioMed Central} and take full advantage of:

- Convenient online submission

- Thorough peer review

- No space constraints or color figure charges

- Immediate publication on acceptance

- Inclusion in PubMed, CAS, Scopus and Google Scholar

- Research which is freely available for redistribution

Submit your manuscript at www.biomedcentral.com/submit
Biomed Central 